\author{
Research Article
}

\title{
The Effect of DMEM and DMEM:F12 Culture Media on the Growth of SH-SY5Y Cells
}

\author{
Aishah Mohammed Izham ${ }^{1}$, Jasmine Chia Siew Min ${ }^{1}$, Sharmili Vidyadaran ${ }^{1}$, Mohd Roslan Sulaiman ${ }^{1}$, B. \\ Hemabarathy Bharatham ${ }^{2}$ and Enoch Kumar Perimal ${ }^{1}$,*
}

\begin{abstract}
1 Department of Biomedical Science, Faculty of Medicine and Health Sciences, Universiti Putra Malaysia, 43400 Serdang, Selangor Darul Ehsan, Malaysia.
2 Department of Biomedical Sciences, School of Diagnostic and Applied Sciences, Faculty of Health Sciences, Universiti Kebangsaan Malaysia, Jalan Raja Muda Abdul Aziz, 50300 Kuala Lumpur, Malaysia
\end{abstract}

https://doi.org/10.28916/lsmb.2.3.2018.23

Received 7 September 2018, Revisions received 28 November 2018, Accepted 29 November 2018, Available online 31 December 2018

\begin{abstract}
The human neuroblastoma cell line, SH-SY5Y cells, derived from the parental SK-N-SH cell line, is commonly used as an in vitro model for neuroscience and neurobiology research. Since SH-SY5Y cells are widely cultured for research, several different culture media have been used to optimize the growth of the cells, including Eagle's Minimum Essential Medium (EMEM), Dulbecco's modified Eagle's medium (DMEM) and other recently developed culture media. SH-SY5Y cells has the ability to reach confluency in culture flasks ranges from 5 days to 15 days, depending on the culture media used. Hence, the optimization of the culture media is crucial to achieve the fastest growth rate for the cells. The objective of the study is to evaluate the culture media for the proliferation of SH-SY5Y cells. We compared the growth rate of SH-SY5Y cells cultured in Dulbecco's modified Eagle's medium (DMEM) supplemented with 15\% heat-inactivated fetal bovine serum (hiFBS), Dulbecco's modified Eagle's medium: Nutrient mixture F-12 (DMEM:F12) + supplemented with 15\% hiFBS and DMEM:F12 supplemented with 10\% hiFBS. In DMEM:F12 supplemented with 15\% hiFBS, cells grew up to 6.67E+05 cells. In DMEM:F12 supplemented with $10 \%$ hiFBS, cells grew up to $5.28 \mathrm{E}+05$ cells. In DMEM supplemented with $15 \%$ hiFBS, the cells grew up to $4.76 \mathrm{E}+05$ cells. There was a significant difference between culture media DMEM:F12 supplemented with 15\% hiFBS as compared to DMEM:F12 supplemented with $10 \%$ hiFBS and DMEM supplemented with $15 \%$ hiFBS $(p<0.05)$ following $24 \mathrm{~h}$ incubation. However, there is no significant difference between DMEM:F12 supplemented with 10\%hiFBS as compared to DMEM supplemented with $15 \%$ hiFBS $(p>0.05)$. We found that DMEM:F12 supplemented with $15 \%$ hiFBS could serve as an optimized culture media for high proliferation rate of SH-SY5Y cells.
\end{abstract}

Keywords: SH-SY5Y cells; growth rate in vitro; Dulbecco's modified Eagle's medium (DMEM); DMEM:F12; Heat-inactivated fetal bovine serum (hiFBS)

\subsection{Introduction}

The study of the brain and nervous system has been challenging due to the complexity of neuronal cell structure, function and the supporting glial cells. The development of in vitro model of neuronal and glial cell culture has been an important foundation in the research of neurobiology and neurological disorder. The understanding of receptors and channels, neuron specific protein, signal transmission and molecular mechanism could be achieved through in vitro model of the nervous system. In order to create the in vitro model, primary cells, such as adult human neurons as well as primary neurons from rodents, were used (Hattangady and Rajadhyaksha, 2009). The constraint of using primary cells is the limited capacity of proliferation. Besides, adult human neurons are not readily available. Hence, alternative transformed cell lines were established to overcome the limitation. Several cell lines have been commonly used in neuroscience research, such as human neuroblastoma SH-SY5Y cells, mouse neuroectodermal NE4C cells, mouse neuroblastoma Neuro-2a cells and rat pheochromocytoma PC-12 cells (Forster et al., 2016). These cells were used in neurobiology studies due to their similar morphology and physiology to adult human neuronal cell, albeit there are some distinct differences in rodents' cells and cancerous cells. Since the culture of primary cells would cause problems in replication and the level of protein yield, SH-SY5Y, a human neuroblastoma cell line, have been utilized to study neuropathic pain as well as other neurological condition and its prophylactics (Dwane et al., 2013; Popova et al., 2017).

SH-SY5Y cells, a human neuroblastoma cell line, was first derived from the parental cell line SK-N-SH obtained through a bone marrow biopsy in 1970 (Shipley et al., 2016). SH-SY5Y cell culture encompasses of adherent and floating cells, whereby most neurobiology researches use the adherent cells. Another characteristic of SH-SY5Y cells is its ability to be differentiated to a mature neuron, which expresses mature marker and phenotype. Differentiated SH-SY5Y cells exhibit phenotype such as neurite outgrowth, the expression of cholinergic,

\footnotetext{
* Correspondence: Department of Biomedical Science, Faculty of Medicine and Health Sciences, Universiti Putra Malaysia, 43400 Serdang, Selangor Darul Ehsan, Malaysia

e-Mail: enoch@upm.edu.my
}

(C) 2018 by the Author(s). Life Sciences, Medicine and Biomedicine (ISSN: 2600-7207) Published by Biome Journals. Wholly owned by Biome Scientia Sdn Bhd., previously by WWH Networks Sdn Bhd. Attribution-ShareAlike 4.0 International (CC BY-SA 4.0). This open access article is distributed based on the terms and conditions of the Creative Commons Attribution license https://creativecommons.org/licenses/by-sa/4.0/ 
dopaminergic and adrenergic receptors and neurone-specific enolase (Xicoy et al., 2017). It was reported that undifferentiated SH-SY5Y cells have neuroblast-like morphology with short neurites while differentiated SH-SY5Y cells are denoted morphologically by extended neurites (SH-SY5Y ATCC ® CRL-2266 ${ }^{\mathrm{TM}}$ ) (Shipley et al., 2016).

The culture media for SH-SY5Y cells varied across researchers, whereby the commonly used are Dulbecco's Modified Essential Medium (DMEM) (Kovalevich and Langford, 2013), DMEM/Ham's F-12 (Murillo et al., 2017) and Eagle's Minimum Essential Medium Eagle (MEM) (Smerker, 2014), with the supplementation of heat inactivated fetal bovine serum (FBS) and antibiotics (SH-SY5Y ATCC ${ }^{\circledR}$ CRL-2266 ${ }^{\mathrm{TM}}$ ). These different culture media formulation differs in terms of the types and amount of amino acids and the amount of inorganic salts present. DMEM contains double the amount of nonessential amino acids as compared to EMEM (Arora, 2013). The combination of DMEM and Ham's:F12 forming DMEM:F12 culture media provides highly enriched supply of amino acids, vitamins and trace elements, hence, suggested to promote the growth of cells (Arora, 2013; Nacalai Tesque Inc., 2014). Furthermore, there are distinct effects of these culture media on cell attachment and the inoculated number of cells for SH-SY5Y and PC12 cells (Smerker, 2014; Sakagami et al., 2017). Besides that, the use of different culture media, such as DMEM and RPMI-1640 (developed by Roswell Park Memorial Institute), could also influence the differentiation capacity of the cell lines and the production of metabolome of the cells (Xicoy et al., 2017). The concentration of inorganic salts, such as sodium pyruvate, within the culture media also determine the ability of the media to protect the cell lines from oxidative stress. Thus, it is crucial to choose the most suitable culture media for the cell lines. The aim of our study is to optimize the protocol that provides the most advantageous growth for SH-SY5Y cells.

\section{Materials and methods}

\section{$2.1 \quad$ Reagents}

Dulbecco's Modified Essential Medium (DMEM), Dulbecco's Modified Essential Medium/Ham's Nutrient Mixture (DMEM:F12), Penicillin-Streptomycin solution and 2.5g/l-Trypsin/1mmol/l-EDTA Solution were purchased from Nacalai Tesque (Tokyo, Japan). Fetal bovine serum (FBS) was purchased from Gibco-BRL (Grand Island, NY). Phosphate buffered saline (PBS) were purchased from Goldbio (St. Louis, MO).

\section{$2.2 \quad$ Cell culture}

The SH-SY5Y neuroblastoma cells were given by Dr. Say Yee How from the Department of Biomedical Sciences, Universiti Tunku Abdul Rahman. The cells were initially grown to confluence in T-25 flasks in Dulbecco's Modified Essential Medium (DMEM) which contains $4.5 \mathrm{~g} / \mathrm{l}$ glucose with $2 \mathrm{mM}$ of L-glutamine and sodium pyruvate, supplemented with $15 \%$ heat inactivated fetal bovine serum (hiFBS) and $1 \%$ of Penicillin-Streptomycin mixed solution at $37^{\circ} \mathrm{C}$ with $5 \%$ carbon dioxide $\left(\mathrm{CO}_{2}\right)$.

The cells were plated at $2 \times 10^{5}$ cells/well in 24 well-plates. Three different media compositions were used to culture the cells which are DMEM:F12 with 15\% hiFBS, DMEM:F12 with 10\% hiFBS and DMEM with 15\% hiFBS. All cultures were supplemented with 1\% of Penicillin-Streptomycin mixed solution and incubated at at $37^{\circ} \mathrm{C}$ with $5 \%$ carbon dioxide.

\subsection{Determination of viable cells}

Following 24hours of incubation, the cells were observed under the microscope and the cell viability was examined by using the hemocytometer. The number of cells was demonstrated in Figure 4. Images of the cells under 10X magnification were captured to observe confluency and the morphology.

\subsection{Statistical analysis}

The results analysis was conducted through the one-way analysis of variance (ANOVA) followed by Tukey's post hoc test. The significance level was set at $p<0.05$.

\subsection{Results}

\subsection{Effect of the different culture media on the cell morphology}

The morphology of the cells incubated in three different culture media - (a) DMEM:F12 with 15\% hiFBS, (b) DMEM:F12 with 10\% hiFBS and (c) DMEM with 15\% hiFBS were also evaluated. Following 24 hours of incubation, cells grown in all three culture media remained in the innate undifferentiated state (Figure 1).
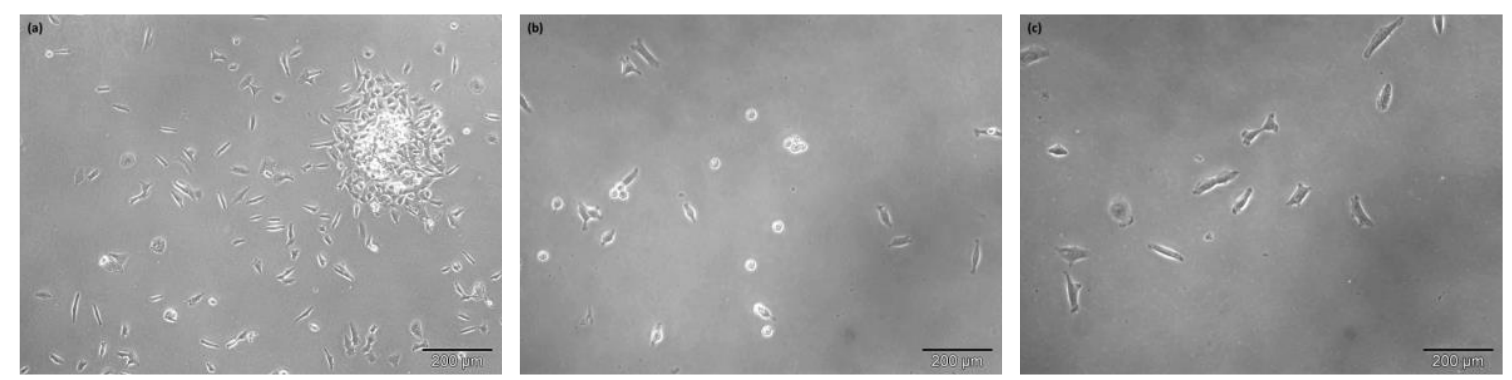

Figure 1: Microphotographs of SH-SY5Y cells in different culture media. Cells were plated on 24 well-plates uncoated, incubated at $37^{\circ} \mathrm{C}$ with $5.0 \%$ carbon dioxide. Pictures were taken at $10 \mathrm{X}$ using Cell Signal software. Scale bar $=200 \mu \mathrm{m}$. (a) SH-SY5Y cells cultured in DMEM:F12 supplemented with 15\% hiFBS 10X magnification. (b) SH-SY5Y cells cultured in DMEM:F12 supplemented with 10\% hiFBS at 10X magnification. (c) SH-SY5Y cells cultured in DMEM supplemented with 10\% hiFBS at 10X magnification. It can be observed that cells in (a) showed the highest number followed by cells in (b) and (c). 
The growth rate of SH-SY5Y cells was evaluated in three varying culture media, presented in Figure 2. The seeding numbers for the cells were 2.0E+05 cells in all the three media. In DMEM:F12 supplemented with 15\% hiFBS, cells grew up to 6.67E+05 cells. In DMEM:F12 supplemented with $10 \%$ hiFBS, cells grew up to 5.28E+05 cells. In DMEM supplemented with 15\% hiFBS, the cells grew up to $4.76 \mathrm{E}+05$ cells. There was a significant difference $(p<0.05)$ in the cell growth of SH-SY5Y cells between culture media DMEM:F12 supplemented with 15\% hiFBS as compared to DMEM:F12 10\%hiFBS and DMEM 15\% hiFBS. There is no significant difference $(p>0.05)$ between DMEM:F12 supplemented with $10 \%$ hiFBS as compared to DMEM 15\% hiFBS.

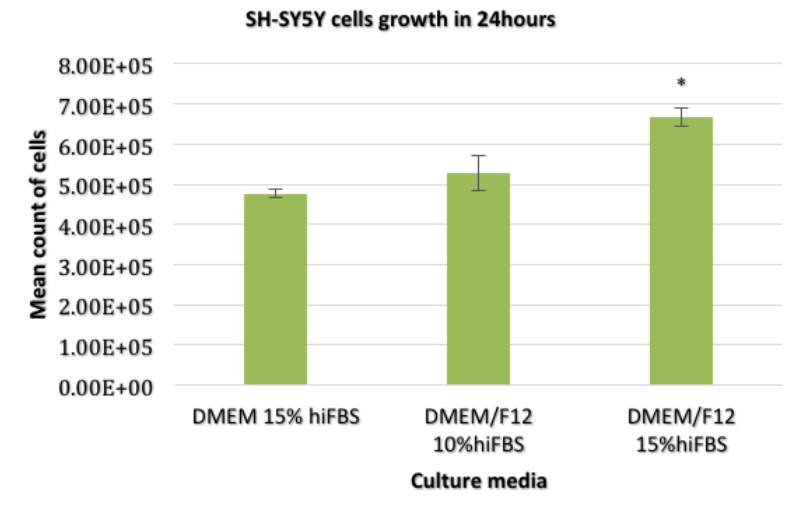

Figure 2: Quantitative analysis of number of SH-SY5Y cells in different culture media. The cells were counted after $24 \mathrm{~h}$ of incubation following initial plating and the average number of cells for each culture media was presented as mean values $\pm S D, n=3$. Bars represent the mean cell count of cells from three experiments. Significant differences were measured through the oneway analysis of variance (ANOVA) followed by Tukey's post hoc test. * $p<0.05$ in comparison to DMEM $15 \%$ hiFBS and DMEM/F12 10\%hiFBS.

\section{0}

\section{Discussion}

From the results shown, the morphology of SH-SY5Y cells appeared to be flat, large with neuroblast-like morphology. This is also observed in other experiments on undifferentiated SH-SY5Y cells (Kovalevich and Langford, 2013; Shipley et al., 2016). Similar morphology was documented by The American Type Culture Collection for SH-SY5Y cells, suggesting that DMEM and DMEM:F12 culture media did not alter the morphology of the cells. In this experiment, we observed that the growth rate of SH-SY5Y cells was the fastest in DMEM:F12 culture media with the supplementation of 15\% hiFBS compared to cells cultured in DMEM:F12 with 10\% hiFBS and DMEM with 15\% hiFBS. This could be due to the enriched formulation of DMEM:F12 which is higher in amino acids (with the addition of L-Isoleucine, Lglutamic acid, L-Proline, L-Aspartic acid, L-Cystine- $\mathrm{HCl}-\mathrm{H}_{2} \mathrm{O}$, L-Alanine and L-Asparagine- $\mathrm{H}_{2} \mathrm{O}$ ) as well as inorganic salts, as compared to DMEM culture media (Nacalai Tesque, 2014).

Although there was no statistically significant difference between DMEM:F12 supplemented with 10\% hiFBS and DMEM supplemented with $15 \%$ hiFBS, the cells in the former culture media showed higher number following $24 \mathrm{~h}$ of incubation. Similar results were observed in a study which showed the growth of SH-SY5Y cells in DMEM:F12 media was better than in DMEM media following 24 hours of incubation (Sakagami et al., 2017). It was also reported that SH-SY5Y cells consumed Glutamine the highest (present in both culture media), followed by Isoleucine and Serine (enhanced in DMEM:F12 media) (Sakagami et al., 2017). Glutamine is profuse in the human blood for its role neuroprotective role against DNA damage and reactive oxidative stress. It is also essential for $N$-methyl-D-aspartate (NMDA) receptors, which is important for the physiology of neuronal cells (Chen and Herrup, 2012). Apart from that, isoleucine is suggested to be important in ammonia detoxification within the human brain environment through the catabolism of the amino acid to tricarboxylic acid (TCA) (Bak et al., 2009). This explains the significant difference of SH-SY5Y cells growth in DMEM:F12 and DMEM.

The concentration of hiFBS supplemented to DMEM:F12 culture media had also shown to exhibit effect on the growth period of SH-SY5Y cells. Higher concentration of hiFBS had shown to cause a faster growth in SH-SY5Y cells. This could suggest that the presence of growth factors within the serum in addition to the essential amino acids present in DMEM:F12 enhance the proliferation of the cells within 24 hours (Shipley et al., 2016; Fang et al., 2017). Comparable result were observed in rabbit limbal stem cells which shown to have the highest number of cells after 14 days of cultivation when cultured in DMEM:F12 supplemented with 16\% FBS as compared to DMEM supplemented with other types of serum or cell feeder (Gürdal et al., 2018).

\section{0}

\section{Conclusion}

Our present findings will provide better understanding into future usage of a more suitable culture media in relation of SH-SY5Y cell growth. In conclusion, from this study we found that DMEM:F12 supplemented with 15\% hiFBS could serve as an optimized culture media for high proliferation rate of SH-SY5Y cells. Further studies should be conducted with more variables of incubation time and the consumption of the amino acids within the culture media to observe the effects on SH-SY5Y cells growth.

\section{0}

\section{Conflict of Interest}

The authors declare no conflict of interest.

\section{Acknowledgements}

This study was supported by Geran Putra Berimpak (UPM/800-3/3/1/GPB/2018/9659000) by Universiti Putra Malaysia. The authors are thankful to the Faculty of Medicine and Health Sciences, Universiti Putra Malaysia, and the Physiology Laboratory for providing the necessary support for the study. 
Bak, L. K., Iversen, P., Sørensen, M., Keiding, S., Vilstrup, H., Ott, P., Waagepetersen, H. S., \& Schousboe, A. (2009). Metabolic fate of isoleucine in a rat model of hepatic encephalopathy and in cultured neural cells exposed to ammonia. Met Brain Dis, 24(1), 135-145.

Chen, J., \& Herrup, K. (2012). Glutamine acts as a neuroprotectant against DNA damage, beta-amyloid and H2O2-induced stress. PLoS One, $7(3), \mathrm{e} 33177$

Dwane, S., Durack, E., \& Kiely, P. A. (2013). Optimising parameters for the differentiation of SH-SY5Y cells to study cell adhesion and cell migration. BMC Res Notes, 6, 366.

Fang, C. Y., Wu, C. C., Fang, C. L., Chen, W. Y., \& Chen, C. L. (2017). Long-term growth comparison studies of FBS and FBS alternatives in six head and neck cell lines. PLoS One, 12(6), e0178960.

Forster, J. I., Koglsberger, S., Trefois, C., Boyd, O., Baumuratov, A. S., Buck, L., Balling, R., \& Antony, P. M. (2016). Characterization of differentiated SH-SY5Y as neuronal screening model reveals increased oxidative vulnerability. J Biomol Screen, 21(5), $496-509$.

Gürdal, M., Barut Selver, Ö., Baysal, K., \& Durak, İ. (2018). Comparison of culture media indicates a role for autologous serum in enhancing phenotypic preservation of rabbit limbal stem cells in explant culture. Cytotechnology, 70(2), 687-700.

Hattangady, N. G., \& Rajadhyaksha, M. S. (2009). A brief review of in vitro models of diabetic neuropathy. Int J Diabetes Dev Ctries, 29(4), 143-149.

Kovalevich, J., \& Langford, D. (2013). Considerations for the use of SH-SY5Y neuroblastoma cells in neurobiology. Neuronal cell culture (pp. 9-21): Springer.

Popova, D., Karlsson, J., \& Jacobsson, S. O. P. (2017). Comparison of neurons derived from mouse p19, rat pc12 and human SH-SY5Y cells in the assessment of chemical- and toxin-induced neurotoxicity. BMC Pharmacol Toxicol, 18(1), 42.

Sakagami, H., Suzuki, R., Shirataki, Y., Iwama, S., Nakagawa, M., Suzuki, H., Tanaka, K., Tamura, N., \& Takeshima, H. (2017). Re-evaluation of culture condition of pc12 and SH-SY5Y cells based on growth rate and amino acid consumption. In Vivo, 31(6), 1089-1095.

Shipley, M. M., Mangold, C. A., \& Szpara, M. L. (2016). Differentiation of the SH-SY5Y human neuroblastoma cell line. J Vis Exp(108), 53193.

Smerker, H. (2014). Weaning and adaptation of SH-SY5Y cells to low glucose media for manganese exposure studies. 2014 NCUR.

Xicoy, H., Wieringa, B., \& Martens, G. J. (2017). The SH-SY5Y cell line in Parkinson's disease research: A systematic review. Mol Neurodegener, 12(1), 10. 